\title{
Bullying entre adolescentes em Sergipe: Estudo na Capital e Interior do Estado
}

\author{
Luana Cristina Silva Santos'; https://orcid.org/0000-0001-7972-0366 \\ André Faro1; https://orcid.org/0000-0002-7348-6297
}

\begin{abstract}
Resumo
Esta pesquisa objetivou conhecer a distribuição social do bullying em uma amostra de adolescentes no Estado de Sergipe. Participaram 555 sujeitos entre 14 e 18 anos. Utilizou-se a Escala Califórnia de Vitimização ao Bullying (ECVB) e um questionário sociodemográfico. Realizouse regressão logística (método Forward Wald), tendo como variável dependente o bullying e variáveis independentes as relativas ao perfil sociodemográfico. Dentre os participantes, $19,1 \%(n=106)$ foram classificados como vítimas de bullies. O modelo de regressão classificou como preditores do bullying o tipo de escola [OddsRatio $(O . R)=2,$.8 para escolas privadas], município $(O . R .=1,8$ para a capital) e idade $(O . R .=1,9$, para 16 anos; 2,0 para até 17 anos; 1,3 para mais de 17 anos). Ao final foram recomendados novos estudos a respeito da predição de variáveis, visto que tipo de escola e município, por exemplo, ampliaram as chances de vitimização ao bullying.
\end{abstract}

Palavras-chave: Adolescentes; bullying; escola.

\section{Bullying among adolescents in Sergipe: Study in the State Capital and Interior}

\begin{abstract}
This research aimed to know the social distribution of bullying in a sample of adolescents in the State of Sergipe. 555 subjects between 14 and 18 years old participated. The California Scale of Bullying Victimization (ECVB) and a sociodemographic questionnaire has used. Logistic regression was performed (Forward Wald method), with as dependent variable the bullying and independent variables as those related to the sociodemographic profile. Among the participants, $19.1 \%(n=106)$ were classified as victims of bullies. The regression model classified as predictors of bullying the type of school [OddsRatio $(O R)=2.8$ for private schools], municipality $(\mathrm{OR}=1.8$ for capital) and age $(\mathrm{OR}=1.9$ for 16 years 2.0 for up to 17 years, 1.3 for over 17 years). At the end, new studies have recommended regarding the prediction of variables, since type of school and municipality, for example, increased the chances of victimization to bullying.
\end{abstract}

Keywords: Adolescents; bullying; school.

\section{Bullying entre adolescentes en Sergipe: Estudio en la Capital e Interior del Estado}

\section{Resumen}

En esta investigación se tuvo por objetivo conocer la distribución social del bullying en una muestra de adolescentes en el Estado de Sergipe. Participaron 555 sujetos entre 14 y 18 años. Se utilizó la Escala California de Victimización al Bullying (ECVB) y un cuestionario sociodemográfico. Se realizó regresión logística (método Forward Wald), teniendo como variable dependiente el bullying y variables independientes las relativas al perfil sociodemográfico. Entre los participantes, el 19,1\% $(n=106)$ fueron clasificados como víctimas de bullies. El modelo de regresión clasificó como predictores del bullying o tipo de escuela [OddsRatio $(O . R)=2,$.8 para escuelas privadas], municipio $(O . R$. = 1,8 para la capital) y edad $(O . R .=1,9$, para 16 años; 2,0 para hasta 17 años; 1,3 para más de 17 años). Al final se recomendó nuevos estudios a respeto de la predicción de variables, visto que tipo de escuela y municipio, por ejemplo, ampliaronlas oportunidades de victimización al bullying.

Palabras clave: Adolescentes; bullying; escuela.

1 Universidade Federal de Sergipe - Aracaju - SE - Brasil; luusilva_psy@hotmail.com; andrefaro@ufs.com.br 


\section{Introdução}

Há cerca de 20 anos, a estimativa da prevalência do bullying era de aproximadamente 15\% entre sujeitos de 7 a 16 anos (Olweus, 1996). Atualmente, essa estimativa varia entre 7 e 52\%, a depender do território pesquisado (Silva \& cols., 2018; United Nations Children's Fund [UNICEF], 2014). No Brasil, a expectativa é de que pelo menos $13 \%$ dos adolescentes sofram algum tipo de bullying ao longo da vida (UNICEF, 2014). Apesar de ser considerado um fenômeno antigo, o estudo sistemático do bullying é recente, a partir de 1970, quando o professor e pioneiro no assunto, Dan Olweus, iniciou suas pesquisas e propostas de intervenção (Rigby, 2007).

O fenômeno do bullying é conceituado como um comportamento de opressão, física ou psicológica, repetitivo e sistemático, de um indivíduo ou grupo que se percebe como mais poderoso, para um indivíduo ou grupo percebido como menos poderoso, sem qualquer justificativa para tal comportamento (Fontes, 2013; Olweus, 1996). Além disso, é comumente visto como um processo cíclico no qual o indivíduo ou grupo que é percebido como uma vítima potencial por outro ou por um grupo "mais poderoso" sofre alguma agressão, humilhação ou rebaixamento de forma direta ou indireta, repetidamente (Rigby, 1999).

O bullying se classifica em físico, verbal, relacional e eletrônico, sendo o físico o tipo mais fácil de observar (Bandeira, 2009). O tipo físico envolve socos, chutes, pontapés, roubo do lanche e, segundo estudos, a tendência é que sua frequência diminua com a idade (Berger, 2007; Rolim, 2008). O verbal inclui práticas que envolvem insultar ou atribuir apelidos vergonhosos como comentários racistas, homofóbicos ou de diferenças socioeconômicas, por exemplo, e tende a ser mais comum que o tipo físico com o aumento da idade (Berger, 2007). A forma relacional do bullying caracteriza-se por afetar o relacionamento da vítima com seus colegas e se torna mais comum com o início da puberdade e fortalecimento da cultura dos pares (Berger, 2007). Por fim, o bullying eletrônico (ou cyberbullying, como tem sido denominado), envolve as agressões por vias eletrônicas e sua prevalência tem crescido com o aumento do contato de crianças com a internet, especialmente redes sociais (Kowalski \& Limber, 2007; Rivers, Chesney, \& Coyne, 2011).

O cenário típico do bullying é a escola, local onde ocorre a maior parte do contato das crianças e adolescentes com pares de mesma idade (Alves, 2015; Bandeira, 2009). Os papéis em geral se dividem em: vítima, agressor, vítima/ agressor e testemunhas. O agressor é o autor, aquele que se percebe como mais forte que alguém ou um grupo e direciona a eles ações agressivas; a vítima é o alvo exposto a repetidas agressões as quais não tem força (real ou percebida) para revidar (Berger, 2007); a vítima/agressor é aquela que desempenha os dois papéis, de vítima e de agressor (Roland, 2002) e a testemunha é o indivíduo que presencia o bullying, mas não está no papel de vítima ou agressor (Berger, 2007).

Alguns estudos classificam os agressores como mais passíveis a engajar-se em comportamentos de risco futuramente, como consumir tabaco ou álcool, assim como em comportamentos antissociais (Lopes Neto, 2005). Já estudos sobre as vítimas mostram que elas parecem possuir características que as tornam vulneráveis, como possuir poucos amigos, ser passivo, retraído e possuir baixa autoestima (Catini, 2004). Além das consequências imediatas e diretas, tem-se pesquisado consequências do bullying a longo prazo, as quais envolvem sintomatologia psicológica e fisiológica, tais como enurese noturna, alterações do sono, cefaleias, dores epigástricas, isolamento, irritabilidade, depressão, pânico e, inclusive, atos deliberados de autoagressão (Heilbron \& Prinstein, 2010; Lopes Neto, 2005). Os indivíduos que sofrem bullying são, em geral, mais ansiosos e inseguros que os outros estudantes, além de frequentemente reagirem ao ataque de forma passiva, chorando ou retirando-se somente, visto não serem pessoas agressivas ou provocativas (Olweus, 1996; Rigby, 2007). Além disso, dado este padrão de reação submisso e acuado, o fenômeno torna-se de difícil identificação, já que depende da denúncia ativa da vítima, que deve estar exposta repetidamente a ações negativas e que trazem desconforto por parte de outros estudantes (Bandeira, 2009; Olweus, 1996).

A literatura mostra relação inversa entre idade $e$ bullying, sendo maior a magnitude do risco para alunos mais novos (Malta \& cols., 2014), tanto internacionalmente (como encontrado no estudo de Melim e Pereira, 2013, por exemplo), quanto nacionalmente (Santos, Cabral-Xavier, Paiva, \& Leite-Cavalcanti, 2014), e, além disso, essa relação parece estar sendo mantida desde os estudos pioneiros do fenômeno (Olweus, 1993). Em geral, são os meninos que mais sofrem (Malta \& cols., 2014; Santos \& cols., 2014) e praticam bullying (Rech, Halpern, Tedesco, \& Santos, 2012), não sendo comum encontrar diferenças significativas entre os sexos (Bandeira, 2009). Entretanto, alguns estudos mostram vulnerabilidade semelhante para ambos os sexos (Melim \& Pereira, 2013). Apesar da heterogeneidade de achados, a literatura mostra que alguns fatores parecem funcionar como proteção para os adolescentes em relação à exposição ao bullying, como religiosidade (Resnick, Ireland, \& Borowsky, 2004) e suporte social (Rothon, Head, Klineberg, \& Stansfeld, 2011), por exemplo.

Apesar de o bullying ocorrer principalmente na escola, atinge toda a coletividade de atores sociais participantes da comunidade educativa em torno do aluno, como família, educadores, equipes técnicas e pares (Centro de Empreendedorismo Social e Administração em Terceiro Setor [CEATS], 2010). Assim, todos do meio social proximal da vítima podem ser úteis ao planejar ações, identificar necessidades ou mesmo propor soluções, pautando-se no conhecimento do fenômeno e observação da sua ocorrência (Rigby, 2007). Foi Olweus (1996) quem, além de iniciar o estudo do fenômeno, propôs as primeiras medidas para estimativa de sua prevalência, as quais têm sido adaptadas e otimizadas até os dias atuais. Na realidade brasileira ainda se percebe relativa escassez de ferramentas úteis para a avaliação do bullying (Alckmin-Carvalho, Izbicki, Fernandes, \& Melo, 2014). Ademais, os estudos nacionais são em geral realizados nas capitais dos estados, poucos são os que coletam dados também em municípios do interior e mesmos esses 
não objetivam fazer a comparação dos dados entre a capital e municípios do interior (CEATS, 2010).

Na avaliação de um fenômeno como esse é necessário desenvolver e aprimorar medidas sensíveis aos seus principais aspectos, a saber: frequência, intencionalidade e desequilíbrio de poder (Felix, Sharkey, Green, Furlong, \& Tanigawa, 2011). A diferenciação de vitimização por pares e bullying leva em conta exatamente estes três aspectos básicos, considerando o bullying a agressão que ocorre repetitivamente, causando incômodo na vítima que percebe como intencional a ação do agressor e, além disso, possui uma autopercepção de inferioridade em relação ao mesmo. Qualquer tipo de vitimização que não possua essas características é denominado vitimização por pares (Felix \& cols., 2011). Nesse sentido, a Escala Califórnia de Vitimização ao Bullying (ECVB) tem se mostrado pertinente por contemplar os três aspectos do fenômeno, pois permite, além de uma identificação rápida do fenômeno, diferenciar as vítimas de bullies e as vítimas apenas de pares (Soares, Gouveia, Gouveia, Fonsêca, \& Pimentel, 2015). Assim, a classificação da escala permite delineamentos de análise que tornam possível mapear maior vulnerabilidade ao fenômeno do bullying em relação aos aspectos sociodemográficos.

Em suma, acredita-se que para maior eficácia das ações de prevenção do bullying, elas devem ser balizadas pelo conhecimento acerca da prevalência e distribuição social desse fenômeno, o que fora apontado também por diferentes autores na temática (Antunes \& Zuin, 2008; Binsfeld \& Lisboa, 2010; Lopes Neto, 2005; Silva \& cols., 2018). Assim, é favorecido o alcance de maior acurácia no conhecimento de características de sua ocorrência e impacto diferenciado nos grupos mais vulneráveis. Logo, o presente estudo pretendeu investigar a prevalência do bullying em uma amostra de adolescentes de Sergipe, estudantes em escolas da capital e do interior do Estado.

\section{Método}

\section{Participantes}

A amostra foi composta por 555 indivíduos adolescentes entre 14 a 18 anos, residentes em Aracaju (capital, 650.106 habitantes $\left.^{1}\right)(n=282)$ ou em Nossa Senhora da Glória ( $n=273$ ), município do interior do Estado de Sergipe (32.497 habitantes ${ }^{2}$ ). Conduziu-se a estratégia de coleta por conveniência, sendo duas escolas (uma da rede pública e uma da rede privada) em cada local, envolvendo todos os alunos do Ensino Médio $\left(1^{\circ}, 2^{\circ}\right.$ e $3^{\circ}$ anos).

\section{Instrumentos}

Foi utilizada a versão da ECVB desenvolvida por Felix e cols. (2011), adaptada e validada ao contexto brasi-

\footnotetext{
1 Ver https://cidades.ibge.gov.br/brasil/se/aracaju/panorama

2 Ver https://cidades.ibge.gov.br/brasil/se/nossa-senhora-da-gloria/ panorama
}

leiro por Soares e cols. (2015). A ECVB é composta por sete itens que avaliam formas de bullying, respondidas em escala do tipo Likert de cinco pontos (varia entre "nunca" e "várias vezes durante esta semana"). Após cada um dos sete itens existe ainda uma pergunta cuja resposta é nominal (sim ou não): "Este comportamento foi intencional e teve importância para você (o magoou)?", cujo propósito é diferenciar vítimas de bullying e de pares. Ao final, há uma lista com 10 adjetivos para que o respondente faça a comparação entre si e o agressor numa resposta do tipo Likert de três pontos (menos do que eu, parecido comigo, mais do que eu), buscando-se avaliação da percepção do desequilíbrio de poder.

A classificação final da ECVB é obtida pela avaliação dos itens separadamente para cada tabela ou aspecto mensurado, e sua posterior classificação com base no estudo de Felix e cols. (2011). Obtém-se um escore sobre ter sofrido ou não experiências de vitimização, se esta vitimização foi intencional e outro sobre a percepção de desequilíbrio de poder. Classifica-se da seguinte forma: não vítimas são aquelas que não reportam nenhuma experiência de vitimização e desequilíbrio de poder, ou seja, possuem zero no primeiro escore e no terceiro; vítimas de pares relatam pelo menos uma experiência de vitimização a qualquer frequência, mas não percebem desequilíbrio de poder, marcando mais do que zero no primeiro escore e entre zero e 10 no terceiro sem relatar "mais do que eu"; e vítimas de bullies relatam pelo menos um tipo de vitimização especificamente duas a três vezes por mês, que essa vitimização foi intencional pelo menos alguma vez, havendo desequilíbrio de poder, ou seja, pontuar mais que dois no primeiro escore sendo ao menos um item "duas ou três vezes no último mês", o segundo escore deve ser menor que sete, e o terceiro maior que 10 ou haver pelo menos um item de pontuação dois (mais do que eu). Neste estudo, quem não relatou ocorrência de situação nenhuma, mas se sentiu incomodado ou percebeu desequilíbrio de poder foi excluído da amostra, dada a possível inconsistência entre sentir-se incomodado sem o relato da ocorrência de alguma situação (40 casos).

Administrou-se também um questionário sociodemográfico com as variáveis: tipo de escola (pública ou particular), município (capital ou interior), idade (em anos), sexo (masculino ou feminino), escolaridade do pai e escolaridade da mãe (fundamental, médio, superior ou outro, sendo nesta categoria incluídos os não alfabetizados ou que escrevem apenas o nome) e duas perguntas relativas ao suporte social (de zero a '10 ou mais', com quantas pessoas você acha que realmente poderia contar numa situação de extrema dificuldade; e de zero a '10 ou mais', com quantas pessoas você acha que realmente poderia conversar, diante de uma situação de extrema dificuldade).

\section{Procedimentos}

Em observância à legislação em pesquisa com seres humanos, esta pesquisa foi aprovada pela Comissão de Ética e Pesquisa da Universidade Federal de Sergipe (CAAE 52020615.4.0000.5546) e obteve-se previamente a 
autorização da coordenação das escolas. Os participantes assinaram o Termo de Consentimento Livre e Esclarecido, que foi entregue durante a apresentação e explicação da pesquisa. A coleta de dados ocorreu ao longo da semana, de modo coletivo, por meio de um questionário autoaplicável, utilizando-se de 15 minutos de uma aula, mediante consentimento do professore da direção da escola.

\section{Análise de dados}

Inicialmente, conduziram-se procedimentos exploratórios e de ajuste no banco de dados [reposição de missings $(<1 \%)$, tratamento de outliers (<1\%) e erros de digitação], com o auxílio do programa SPSS (versão 20). Para os escores finais das escalas, efetuaram-se as estatísticas descritivas (frequências absoluta e relativa, média e desvio padrão). Em seguida, conduziu-se análise de Regressão Logística (método Forward Wald) para análise de um modelo explicativo da vitimização ao bullying [Variável Dependente (VD)], tendo-se como variáveis explicativas as que compuseram o perfil sociodemográfico (escola, município, idade, sexo, escolaridade do pai, escolaridade da mãe e suporte social). O nível de significância adotado para as análises foi de $p<0,05$.

\section{Resultados}

Dentre os participantes desta pesquisa, foram55,9\% $(n=310)$ do sexo feminino e $44,1 \%(n=245)$ do masculino. A amostra foi composta de $50,3 \%(n=279)$ estudantes de escola pública e 49,7\% ( $n=276$ ) de escola privada, com 50,8\% ( $n$ = 282) na capital do Estado e 49,2\% $(n=273)$ no interior. Em relação à variável escolaridade do pai, a maior parte relatou que este possuía principalmente ensino fundamental $(40,7 \%$; $n=226)$, seguido de ensino médio $(28,8 \% ; n=176)$, ensino superior $(24,5 \% ; n=136)$ e outro $(5,9 \% ; n=33)$ (não alfabetizado ou escreve apenas o nome). Já a distribuição da escolaridade materna foi mais homogênea, com 33,7\% ( $n=187)$ possuindo nível superior, $31,7 \%(n=176)$ nível médio, $29,9 \%$ ( $n=166)$ nível fundamental e 4,7\% $(n=26)$ algum outro nível (não alfabetizado ou apenas escreve o nome).

Os participantes tinham em média 15,6 anos [Desvio Padrão $(D P)=1,22)$ ]. No que diz respeito ao suporte social, pontuaram, em média, 4,8 pontos $(D P=3,01)$ na questão que se referia a quantas pessoas achavam que poderiam contar diante de uma situação de extrema dificuldade e 3,9 pontos $(D P=2,83)$ com relação a quantas pessoas achavam que poderiam conversar diante de uma situação de extrema dificuldade (extremos possíveis em 0 e 10). Dos participantes, $19,1 \%(n=106)$ foram classificados como vítimas de bullies, $61,4 \%$ ( $n=341$ ) eram vítimas de pares e apenas $19,5 \%(n=108)$ classificaram-se como não vítimas.

No sentido de verificar o poder explicativo das variáveis sociodemográficas frente à vitimização bullying $(0=$ não vítimas ou vítimas de pares, $n=449, \mathrm{~F} \%=80,9 ; 1=$ vítimas de bullies, $n=106, F \%=19,1$ ) foi conduzida uma Regressão Logística (Tabela 1), tendo como covariáveis: escola (pública ou privada), município (capital ou interior), idade (até 15 anos, 16 anos, 17 anos e maior que 17 anos), sexo (masculino ou feminino), escolaridade do pai e escolaridade da mãe (fundamental, não alfabetizado ou apenas escreve o nome, médio e superior), suporte social relacionado a quantas pessoas pode-se contar com pessoas numa situação de extrema dificuldade (até 5 e mais que 5) e suporte social relacionado a quantas pessoas pode-se conversar numa situação de extrema dificuldade (até 4 e mais que 4 ).

Tabela 1. Regressão Logística dos Fatores Associados à Vitimização ao Bullying entre Adolescentes estudantes de Sergipe.

\begin{tabular}{llccccc}
\hline Variáveis & & $\boldsymbol{n}(\mathbf{5 5 5})$ & F\% & OR & IC $95 \%$ & $\boldsymbol{P}$ \\
\hline \multirow{2}{*}{ Escola } & Privada & 276 & 49,7 & 2,8 & $1,7-4,5$ & $<0,001$ \\
& Pública & 279 & 50,3 & - & - & - \\
\multirow{2}{*}{ Município } & Capital & 282 & 50,8 & 1,8 & $1,2-2,9$ & 0,007 \\
& Interior & 273 & 49,2 & - & - & - \\
& Até 15 anos & 267 & 48,1 & - & - & - \\
\multirow{2}{*}{ Idade } & 16 anos & 145 & 26,1 & 1,9 & $1,1-3,3$ & 0,015 \\
& Até 17 anos & 98 & 17,7 & 2,0 & $1,1-3,7$ & 0,032 \\
& Maior que 17 anos & 45 & 8,1 & 1,3 & $0,5-3,7$ & 0,634 \\
\hline
\end{tabular}

Notas.

1. $n=$ número de sujeitos; $\mathrm{F} \%$ = indicador de porcentagem; OR = razão de chances (oddsratio); IC 95\% = intervalo de confiança de $95 \% ; p=$ significância estatística.

2. Omnibus test $=7,799 ; p<0,001 ;$ NagelkerkeR ${ }^{2}=0,081(8,1 \%) ;$ Hosmer and Lemeshow Test $=6,912 ; p=0,329$.

3. Variáveis excluídas no modelo final $(p>0,05)$ : Sexo, Escolaridade do Pai, Escolaridade da Mãe e Suporte Social. 
O modelo final foi alcançado em três passos $(-2 L L$ inicial $=527,162$; final $=512,465)$, de modo que permaneceram três variáveis: escola, município e idade.A solução final do modelo revelou que $81 \%$ dos casos foram corretamente preditos (Tabela 1).

Constatou-se que em relação à idade o risco foi amplificado: em comparação aos sujeitos de até 15 anos, os que possuíam 16 (O.R. =1,9; $p=0,015)$ ou 17 anos (O.R. $=2,0 ; p=0,032$ ) tinham aproximadamente duas vezes mais chances de serem vítimas de bullies. Os participantes moradores da capital exibiram quase duas vezes mais chances $(O . R .=1,8 ; p=0,007)$ de serem vítimas de bullying quando comparados a moradores do interior. Por fim, aqueles que estudavam em escola privada $(O . R .=2,8 ; p<0,001)$ tiveram quase três vezes mais chances de serem vítimas, isso em comparação aos alunos da rede pública.

\section{Discussão}

O presente estudo investigou a prevalência da vitimização ao bullying entre adolescentes da capital e em um município do interior de Sergipe. Para tanto, foi observado que na população estudada, $19,1 \%$ dos sujeitos $(n=106)$ foram classificados como vítimas de bullies. Esse valor foi um pouco acima da estimativa de $13 \%$ de vitimização pelo bullying esperada no Brasil, segundo a UNICEF (2014), porém, dentro da faixa de valores entre $7,2 \%$ e $25,4 \%$ encontrados em outros estudos (Bandeira \& Hutz, 2012; Higuita-Gutiérrez \& Cardona-Arias, 2015; Malta \& cols., 2014; Moura, Cruz, \& Quevedo, 2011; Silva \& cols., 2018).

A prevalência do bullying em adolescentes do Estado de Sergipe foi de quase $20 \%$ dentre 555 participantes, ou seja, 106 participantes que foram classificados como vítimas de bullies. Aqueles que estavam na faixa etária entre 16 e 17 anos, residentes da capital do Estado e estudantes de escola privada possuíram entre duas e três vezes mais chances de serem classificados como vítimas. Isso significa que a vulnerabilidade à vitimização em adolescentes aumentou com a idade, o que não coincide com os achados obtidos na literatura, visto que se espera que haja maior magnitude de risco entre sujeitos menores de 15 anos quando comparados aos de 16 anos ou mais (Malta \& cols., 2014, Merlim \& Pereira, 2013; Santos \& cols., 2014). Importante ressaltar que possuir mais de chances de sofrer bullying em relação a determinado subgrupo é um achado importante que pode contribuir com o planejamento de programas e ações específicas a esses subgrupos de maior vulnerabilidade à vitimização por bullying.

Em um estudo com 1818 adolescentes entre 10 e 18 anos de idade, por exemplo, Melim e Pereira (2013) encontraram que $30,2 \%(n=549)$ da amostra podiam ser classificados como vítima de bullying e, destes, somente $1,2 \%(n=22)$ possuía mais que 15 anos. Outro estudo, com adultos entre 18 e 55 anos que responderam questionários sobre suas memórias de vitimização ao bullying, obteve resultados que mostraram que episódios de bullying eram mais lembrados na faixa de idade entre 11 e 13 anos (Elsea \& Rees, 2001). Com isso, entende-se que a discrepância dos achados possa se dever ao fato de que alunos mais velhos tenham vergonha de relatar serem vítimas de bullying (Rigby, 2007), mesmo em questionários anônimos, o que denota a importância de maior acurácia na investigação deste fenômeno.

Neste estudo, os participantes que residiam na capital possuíam aproximadamente duas vezes mais chances de serem vítimas de bullies do que os participantes residentes do interior. Na literatura não foi encontrado nenhum outro estudo que fizesse a comparação entre uma cidade do interior e uma capital, sendo a maioria dos estudos concentrados em uma cidade específica (Santos \& cols., 2014), comparando capitais (Malta \& cols., 2010) ou que não relataram a prevalência específica em cada uma das cidades coletadas, somente a geral (Malta \& cols., 2014). Assim, possíveis contribuições dos dados aqui detectados se direcionam a identificar quais características evocam maior suscetibilidade a sofrer bullying, criando assim um panorama de grupos ou subgrupos que estejam mais comumente expostos. Ou seja, permitir a identificação das vítimas mais usuais e suas características básicas que as exponham a maior risco de ser vítima.

Em relação à comparação entre o tipo de escola, chama-se atenção para o fato de estudantes de escola privada terem demonstrado quase três vezes mais chances de serem vítimas de bullies que estudantes de escola pública. Os estudos também parecem se concentrar em apenas um tipo de escola (Santos \& cols., 2014; Souza, 2013), porém os que realizaram comparação entre escolas públicas e privadas encontraram resultados semelhantes, com maior prevalência de bullying nas escolas privadas (Malta \& cols., 2014). Ressalta-se que em um dos estudos a prevalência foi maior nas escolas privadas apenas na capital em que também foi realizado este estudo (Malta \& cols., 2010) e nenhum avaliou a exposição entre os subgrupos (maior ou menor entre alunos de determinado tipo de escola). Vale destacar que embora se dê maior ênfase às variáveis que se mostraram preditoras com significância estatística, algumas variáveis que não exibiram diferença significativa chamaram atenção neste estudo - especialmente o sexo. Por esse motivo, a seguir são feitas considerações a respeito de tais variáveis.

Não há um consenso sobre sua influência na vitimização ao bullying nos estudos encontrados na literatura nacional segundo o sexo. Alguns revelam diferenças entre meninos e meninas, sendo os meninos a maioria das vítimas (Bandeira \& Hutz, 2012; Malta \& cols., 2014), ao passo que outros trabalhos relatam não haver distinção de sexo (Melim \& Pereira, 2013). Na presente investigação a distribuição das vítimas de bullies em relação ao sexo ficou similar à encontrada na literatura, com aproximadamente $10 \%$ de diferença entre os sexos (Bandeira \& Hutz, 2012), sendo $44,3 \%$ das vítimas do sexo masculino $(n=47)$ e $55,7 \%(n=$ 59 ) do sexo feminino. Entretanto, ao contrário dos trabalhos nacionais que denotam diferença de sexo com maioria da vitimização direcionada ao sexo masculino, neste estudo 
as meninas foram a maioria das vítimas, o que ocorreu em consonância com outro trabalho realizado anteriormente em Aracaju (Souza \& Silva, 2015).

Outra variável relacionada ao bullying na literatura, ainda que de forma escassa, e que não se mostrou associada neste estudo foi a escolaridade parental. Em estudo realizado por Malta e cols. (2010), a maioria das vítimas de bullies $(34,9 \%)$ possuíam mães com ensino superior completo, além de outros estudos também relatarem influência parental no fenômeno do bullying e comportamentos de risco (Flouri \& Buchanan, 2003; Georgiou, 2008). A relação que se pressupõe é que a responsividade materna estaria positivamente relacionada com ajuste da criança e negativamente com comportamentos agressivos como o bullying (Georgiou, 2008). Porém, exceto no estudo de Malta e cols. (2010), que fez um levantamento em relação a escolaridade parental e vitimização ao bullying (sem correlacionar as variáveis), poucos são os estudos que abordam a influência parental, além de não avaliarem, necessariamente,a escolaridade parental como fator de risco ou proteção.

Em síntese, considerando-se o que se obteve em relação à distribuição social do bullying entre adolescentes de Sergipe, torna-se pertinente evidenciar uma possível contribuição desta pesquisa especialmente no que tange à avaliação da distribuição social do bullying. Sobre isso, vale ressaltar que estudos que caracterizam a distribuição social de fenômenos têm sua relevância na saúde e epidemiologia dada pela coleta de dados que representam uma parcela da população a ser estudada, favorecendo-se, assim, a generalização dos resultados obtidos e construção de ações para monitorização, prevenção e tratamento da população (Viacava, 2012).

Acredita-seque o presente estudo, tendo objetivado investigar a prevalência de um fenômeno relativamente comum, pode contribuir com a identificação de subgrupos específicos da amostra que possuem maior vulnerabilidade à vitimização pelo bullying. Em relação ao local da coleta - capital ou interior - apesar do presente estudo ter encontrado indicadores significativamente maiores para adolescentes residentes do interior, ressalta-se que a comparação aqui realizada foi feita somente entre um município localizado no interior e a capital do Estado, não podendo ser generalizada para todos os seus municípios. De qualquer modo, entende-se que estes achados podem servir como baliza inicial para o investimento em estudos que pormenorizem a ocorrência do fenômeno em cidades de pequeno porte ou localizadas fora de regiões metropolitanas.

\section{Considerações Finais}

O presente estudo permitiu o rastreio da distribuição social do bullying em adolescentes sergipanos por meio de uma escala que favorece o diagnóstico rápido - a ECVB - confirmando a significativa prevalência do fenômeno. O bullying é uma situação estressora vivenciada principalmen- te na adolescência e que, em nível extremo, pode culminar em comportamentos suicidas e condutas autolesivas. Nesse sentido, obter estimativas acerca da distribuição social de comportamentos que trazem danos físicos ou psicológicos às vítimas, a exemplo do bullying é essencial para melhor entendimento da amplitude do problema, o que poderá permitir a alocação de serviços e recursos que monitorem e previnam sua ocorrência. Além disso, este estudo também trouxe contribuições importantes ao traçar um perfil de características que ampliam as chances de sofrer vitimização pelo bullying, tais como a avaliação do fenômeno na Capital e interior do Estado, assim como se a escola é particular ou pública.

Como limitações desta pesquisa, julga-se importante considerar que este estudo teve distribuição não-probabilística (ainda que conte com uma amostra relativamente ampla), o que limita a capacidade de generalização das informações então obtidas. Outro aspecto é que ele foi realizado em apenas um município do interior do Estado, não necessariamente refletindo a diversidade socioeconômica de Sergipe como um todo.

Enfim, julga-se pertinente sugerir que novos estudos sejam conduzidos em relação à ocorrência do bullying, principalmente com o intuito de investigar as associações aqui encontradas se repetem, a exemplo entre subgrupos específicos de faixa etária, tipo de escola e município. Tal replicação permitiria aprofundar o conhecimento acerca da vulnerabilidade de subgrupos que podem estar mais suscetíveis a vivenciar e sofrer consequências deletérias - seja no papel de perpetrador, de vítima ou de testemunha - do bullying.

\section{Referências}

Alckmin-Carvalho, F.; Izbicki, S.; Fernandes, L. F. B.; Melo, M. H. S. (2014). Estratégias e instrumentos para a identificação de bullying em estudos nacionais. Avaliação Psicológica, 13(3), 343-350.

Alves, C. F. (2015). Bullying: Gestão escolar e a saúde pública, uma revisão da literatura. Revista Eletrônica Gestão e Saúde, 6(3), 2919-2933.

Antunes, D. C.; Zuin, A. A. S. (2008). Do bullying ao preconceito: Os desafios da barbárie à educação. Psicologia e Sociedade, 20, 3342.

Bandeira, C. M. (2009). Bullying: Autoestima e diferenças de gênero. Dissertação de mestrado, Instituto de Psicologia, Universidade Federal do Rio Grande do Sul, RS.

Bandeira, C. M.; Hutz, C. S. (2012). Bullying: Prevalência, implicações e diferenças entre os gêneros. Psicologia Escolar e Educacional, 16, 35-44.

Berger, K. S. (2007). Update on bullying at school: Science forgotten? Developmental Review, 27, 91-92. 
Binsfeld, A. R.; Lisboa, C. S. M. (2010). Bullying: Um estudo sobre papéis sociais, ansiedade e depressão no contexto escolar do sul do Brasil. Interpersona, 4, 74-105.

Catini, N. (2004). Problematizando o "bullying" para a realidade brasileira. Tese de doutorado, Pontifícia Universidade Católica, Campinas, São Paulo.

Centro de Empreendedorismo Social e Administração em Terceiro Setor [CEATS] (2010). Relatório de Pesquisa "Bullying escolar no Brasil" (Relatório de pesquisa). São Paulo, SP.

Eslea, M.; Rees, J. (2001). At what age children most likely to be bullied at school? Aggressive Behavior, 27, 419-429.Doi: 10.1002/ ab.1027.

Felix, E. D.; Sharkey, J. D.; Green, J. G.; Furlong, M. J.; Tanigawa, D. (2011). Getting precise and pragmatic about the assessment of bullying: The development of the California Bullying Victimization Scale. Aggressive Behavior, 37, 234-247. Doi: 10.1002/ab.20389.

Flouri, E.; Buchanan, A. (2003). The role of mother involvement and father involvement in adolescent bullying behavior. Journal of Interpersonal Violence, 18(6), 634-644. doi: 10.1177/0886260503251129.

Fontes, R. F. F. (2013). Bullying entre pares em contexto escolar: Investigar e intervir para prevenir. Dissertação de mestrado, Universidade do Porto, Portugal.

Georgiou, S. N. (2008). Bullying and victimization at school: The role of mothers. British Journal of Educational Psychology, 78, 109125. Doi: 10.1348/000709907X204363.

Heilbron, N.; Prinstein, M. J. (2010). Adolescent peer victimization, peer status, suicidal ideation, and nonsuicidal self-injury: Examining concurrent and longitudinal associations. Merril-Palmer Quartely, 56, 388-419. Doi: 10.1353/mpq.0.0049.

Higuita-Gutiérrez, L. F.; Cardona-Arias, J. A. (2015). Variables of family, school and social environment context that determine bullying in adolescents in Medellín, Colombia, 2014. Journal of School Violence,16(1), 68-85. Doi: 10.1080/15388220.2015.1112807.

Kowalski, R. M.; Limber, S. P. (2007). Electronic bullying among middle school students. Journal of Adolescent Health, 41(2007), S22-S30. Doi: 10.1016/j.jadohealth.2007.08.017.

Lopes Neto, A. A. (2005). Bullying - Comportamento agressivo entre estudantes. Jornal de Pediatria, 81 (5 Supl), 164-172.

Malta, D. C.; Prado, R. R.; Dias, A. J. R.; Mello, F. C. M.; Silva, M. A. I.; Costa, M. R.; Caiaffa, W. T. (2014). Bullying e fatores associados em adolescentes brasileiros: Análise da Pesquisa Nacional de Saúde do Escolar (PeNSE 2012). Revista Brasileira de Epidemiologia Suplementar PeNSE 2014, 131-145. doi: 10.190/1809-4503201400050011.
Malta, D. C.; Silva, M. A. I.; Mello, F. C. M.; Monteiro, R. A.; Sardinha, L. M. V.; Crespo, C.; ... Porto, D. L. (2010). Bullying nas escolas brasileiras: Resultados da Pesquisa Nacional de Saúde do Escolar (PeNSE), 2009. Ciência e Saúde Coletiva, 15, 3065-3076. doi: 10.1590/S1413-81232010000800011.

Melim, M.; Pereira, B. (2013). Bullying, gênero e idade. In: Silva, P.; Souza, S.; Neto, I. (Orgs.), O desenvolvimento humano: Perspectivas para o século XXI - Memória, Lazer e Atuação Profissional (pp. 292-316). São Luís: EDUFMA.

Moura, D. R.; Cruz, A. C. N.; Quevedo, L. A. (2011). Prevalência e características de escolares vítimas de bullying. Jornal de Pediatria, 87, 19-23. doi: 10.2223/JPED.2042.

Olweus, D. (1993). Bullying at school: What we know and what we can do. Oxford: Blackwell.

Olweus, D. (1996). Bully/Victim problems at school: Facts and effective interventions - Reclaiming children and youth. Journal of Emotional and Behavioral Problems, 5, 15-22.

Rech, R. R.; Halpern, R.; Tedesco, A.; Santos, D. F. (2012). Prevalência e características de vítimas e agressores de bullying. Jornal de Pediatria, 89(2), 164-170. doi: 10.1016/j.jpedp.2012.09.004.

Resnick, M. D.; Ireland, M.; Borowsky, I. (2004) Youth violence perpetration: What protects? What predicts? Findings from the Nacional Longitudinal Study of adolescent health. Journal of Adolescent Health, 35, 424e1-424e10.

Rigby, K. (1999). What harm does bullying do? In Children and Crime: Victims and Offenders Conference (pp. 1-12). Brisbane, Australia.

Rigby, K. (2007). Bullying in schools and what to do about it. Melborne: Australian Council for Education Research.

Rivers, I.; Chesney, T.; Coyne, I. (2011) Cyberbullying. In: Monks, C. P.; Coyne,I.(Orgs.), Bullying in different contexts (p. 211- 230). New York.

Roland, E. (2002). Aggression, depression, and bullying others. Aggressive Behavior, 28, 198-206. doi: 10.1002/ab.90022.

Rolim, M. (2008). Bullying: O pesadelo da escola, um estudo de caso e notas sobre o que fazer. Dissertação de mestrado, Universidade Federal do Rio Grande do Sul, Porto Alegre.

Rothon, C.; Head, J.; Klineberg, E.; Stansfeld, S. (2011). Can social support protect bullied adolescents from adverse outcomes? A prospective study on the effects of bullying on the educational achievement and mental health of adolescents at secondary schools in East London. Journal of Adolescence, 34, 579-588. doi: 10.1016/j.adolescence.2010.02.007.

Santos, J. A.; Cabral-Xavier, A. F.; Paiva, S. M.; Leite-Cavalcanti, A. (2014). Prevalência e tipos de bullying em escolares brasileiros de 
Silva, J. L.; Mello, F. C. M.; Oliveira, W. A.; Prado, R. R.; Silva, M. A. I.; Malta, D. C. (2018). Vitimização por bullying em estudantes brasileiros: Resultados da Pesquisa Nacional do Escolar (Pense). Texto \& Contexto - Enfermagem, 27(3), 1-10. doi: 10.1590/010407072018000310017

Soares, A. K. S.; Gouveia, V. V.; Gouveia, R. S. V.; Fonsêca, P. N.; Pimentel, C. E. (2015). Escala Califórnia de Vitimização do Bullying (ECVB): Evidências de validade e consistência interna. Temas em Psicologia, 23(2), 481-491. doi: 10.9788/TP2015.2-18.

Souza, J. M. (2013). Bullying: Uma das faces do preconceito homofóbico entre jovens no contexto escolar. Dissertação de
Souza, J. M.; Silva, J. P. (2015). Bullying nas escolas: Conhecer para intervir. In: Parente, C. M. D.; Valle, L. E. L. R.; Mattos, M. J. V. M. (Orgs.), A formação de professores e seus desafios frente às mudanças sociais, políticas e tecnológicas (pp. 144-158). São Paulo: Penso Editora.

United Nations Children's Fund [UNICEF] (2014). Hidden in plain sight: A statistical analysis of violence against children (Relatório de pesquisa). New York, NY.

Viacava, F. (2012). Informações em saúde: A importância dos inquéritos populacionais. Ciência e Saúde Coletiva, 7(4), 607-621.

Apoio Financeiro: CAPES

Recebido: 02 de abril de 2017 Aceito: 13 de setembro de 2018 unrestricted use, distribution and reproduction in any medium, provided the original article is properly cited. 